\title{
Single chamber multiple degree-of-freedom soft pneumatic actuator enabled by adjustable stiffness layers
}

\author{
Junius Santoso ${ }^{1}$, Erik H. Skorina ${ }^{1}$, Marco Salerno ${ }^{2}$, Sébastien \\ de Rivaz ${ }^{2}$, Jamie Paik ${ }^{2}$, and Cagdas D. Onal ${ }^{1}$ \\ ${ }^{1}$ Soft Robotics Laboratory, Worcester Polytechnic Institute, Worcester, \\ Massachusetts, USA \\ ${ }^{2}$ Reconfigurable Robotics Laboratory, Ècole Polytechnique Fédérale de Lausanne, \\ Lausanne, Switzerland \\ E-mail: cdonal@wpi.edu \\ October 2018
}

\begin{abstract}
Soft pneumatic actuators promise simple, adaptable, and safe manipulation, but the nature of pneumatic circuits limits the scalability of this approach. This paper introduces a method to augment the degrees-of-freedom of soft pneumatic actuators without increasing the number of independent pressure sources or complex valving networks. Our method achieves multiple degree-of-freedom actuation from a single soft pneumatic chamber by utilizing adjustable stiffness layers based on a thermoplastic polyurethane shape memory polymer (SMP), which changes stiffness when thermally activated. We incorporate SMP layers into a soft pneumatic actuator, allowing multiple degrees of soft deformation to be achieved from a single pressure source by selective activation of SMP layers. A custom printed circuit board acts as a control unit to modularize and enable closed-loop control of the proposed pneumatic actuator. We validated our proposed system and method by varying the temperature of the SMP layer on one side of the actuator while keeping the other side stable near room temperature and observed an increase in bending angle of the pneumatic actuator as a function of temperature. Furthermore, we connected two actuators in series and demonstrated independent actuation, allowing the actuators to form different shapes with a single pressure source. Our results show that the proposed method can augment the degrees of freedom of soft pneumatic actuators as an alternative to multi-chamber or multi-valve systems.
\end{abstract}

Keywords: soft robotics, shape memory polymer, adjustable stiffness, stretchable heater

\section{Introduction}

Soft actuators provide an alternative to the classical rigid actuation systems [1]. The main properties of soft robots, and soft actuators in general, are that they exhibit 
continuum body motion, are capable of large deformations, and are physically compliant [2]. These unique capabilities of soft actuators have led to the emergence of new potential applications in the fields of medical devices [3, 4, 5], search and rescue [6], and assistive robotics [7]. Soft pneumatic actuators are a class of actuators that use compressed air to provide a wide range of motions while maintaining these properties. They are lightweight and highly customizable, which makes them good candidates towards a use in wearable rehabilitative devices and search and rescue robots. An extensive review of fluid-driven soft devices can be found in $[9,10]$.

While multiple degree-of-freedom (DoF) soft robots have been developed [2, $11,12,13]$, their morphology is often complex and requires multi-tubing and/or multi-valve systems in order to control individual soft actuation chambers. Such pneumatic soft robots are also limited in terms of scalability, and can benefit from modularity. Furthermore, the size of the pneumatic circuit limits the actuators from being miniaturized, which is a desirable characteristic for various surgical robotics and inspection applications.

Multi-DoF soft pneumatic robots typically require as many active channels and valves as there are degrees of freedom. The flexible silicone rubber microactuator developed in [11] is capable of changes in pitch, yaw, and length through inflation of three independent internal chambers. The continuum manipulator OctArm can achieve similar motion but is constructed by arranging three or more McKibben actuators radially for each continuum section [12]. The actuators developed in [2], use two parallel air chambers which are controlled through two input pressures. In our previous work, we developed a fully untethered soft robotic snake with an on-board pressure source and independently controlled valves for each of the bending segments [13].

Researchers have acknowledged the benefits of a modular architecture for soft robots [31, 32, 33, 34, 35, 36], enabling simplified restructuring and control of various robot configurations, increasing system redundancy and robustness to failure, easy scalability of the system. Miniaturization and modularization of pneumatic actuators are very difficult to achieve due to the needs of complex tubing and valve networks for independent control. That is, each additional pneumatic chamber requires its own on-board valve or tubes connecting to an external valve. In this paper, we take a different approach and explore the implementation of a variable stiffness layer to increase the degree of freedom of a soft pneumatic actuator without increasing its pneumatic infrastructure and demonstrate its modular operational characteristics.

Although stiffness control of certain soft structures without employing additional layers is also possible, for example by changing the average pressure of the two chambers without changing the pressure difference between them [2], we will focus on methods that utilize adjustable stiffness layers. The control of variable stiffness layers may be achieved by several physical principles. However, due to its simplicity of integration into a soft body, the dominant approach seems to be electrical heating of variable-stiffness materials $[8,14,15,16,17]$. A promising variable-stiffness material is shape memory polymer (SMP) that can transition between a rigid solid state and elastomeric rubbery 
state. Shape memory polymers enable large strain recovery upon heating. Thus, our work considers the use of SMPs combined with a thin flexible heating element as a variable stiffness layer.

Alternatively, the use of low melting point conductive alloys and conductive propylene-based elastomers for variable stiffness application has also been investigated $[16,21]$. These approaches do not require additional heating elements. However, their operation requires large activation voltage in the range of 100-1000 V. Our approach requires much lower activation voltage for more practical use. Other approaches to achieve controllable stiffness include particle jamming [18] and electrostatic friction $[19,20]$. These approaches enable excellent and rapid stiffness change, but suffer from the need for additional vacuum lines and potential limitations due to large electric fields and dielectric breakdown.

The working principle of the soft pneumatic actuator developed in [8] relies on the capability of controlling the stiffness of two shape memory polymer layers that are embedded in silicone rubber, and which lie in parallel with an elongated air chamber. Pressurized air is injected into the air chamber which generates stress in the longitudinal direction of the SMP layers. The radial deformation of the actuator is constrained using wires that are wrapped around it. Therefore, actuation is controlled by modifying the stiffness of each SMP, as well as the input pressure. The particularity of SMPs is that their stiffness is a function of their temperature, and that they are able to recover high deformations when brought back to their initial temperature. Heating the SMP layers may be achieved through resistive heating.

Adjustable stiffness materials have also found use for different applications in robotics. In [17], researchers present a novel variable stiffness gripper that makes use of shape memory polymers for actuation and pin heaters for the heating element. The gripper, which is inspired by the shape of a human finger, makes use of SMP composites at the joints. By independently heating different SMP joints of the gripper, while applying pressure in a soft tube that runs along its spine, the user is able to control the configuration of the robotic arm. In [15], researchers demonstrated a new concept for variable stiffness materials to support reconfigurable surfaces and structures, which were based on shape memory polymer materials. The presented concept makes use of a laminate of both constant and variable stiffness elements.

Soft actuators commonly undergo high strain deformation and since the stiffness change of the shape memory polymer composite is achieved through temperature variation, there is a need for an embedded stretchable heater with reliable temperature control. While many designs and approaches of electrically conducting components have been investigated, it is still challenging to provide solutions for stretchable, robust, self contained, integrated, soft heaters. Our previous work of flexible metallic heater [8] utilizes a serpentine conductor embedded in SMP. This allowed for reliable heating, but required a tedious manufacturing process.

In [23], resistive heating is achieved through the use of a serpentine channel of liquid-phase gallium-indium-tin alloy, while the authors in [24] incorporate the same 
liquid-phase alloy as conductive fillers into poly(dimethylsiloxane) (PDMS) matrix. Galium-indium-tin alloy was chosen due to its lower off-the-shelf price than the eutectic gallium-indium and superior volumetric conductivity compared to the use of percolating network of conductive particles such as carbon black, silver nanoparticles, and graphene. Films made by deposition of prolate particles such as metal nanowires [25], carbon nanotubes [26], or graphene-coated nanocellulose fibers [27] show good performance, but their processing is complex as extra steps may be required to accommodate interparticle contact and to ensure mechanical integrity and protection. The use of Nichrome heating wire in [28] provides an effective way to modulate the temperature given that the wire routing path is kept simple. We opted to use high structure carbon black as conductive filler due to good reproducibility, preservation of mechanical properties, and miniscule resistivity change under large strains [29].

The contributions of this paper include:

- Stretchable heaters film-deposited on SMP layers,

- Scalable augmentation of the active degrees of freedom of soft pneumatic actuators with minimal increase in complexity, and

- Modular multi-DoF soft pneumatic actuation for use in series.

The paper is organized as follows. In the next section, the mathematical model of the pneumatic actuator is introduced, followed by the methods for the manufacturing of the actuator and the design of its control system. Then, the performance of the heating layer is evaluated and the experimental results of a single chamber multiDoF soft pneumatic actuator prototype are presented. Furthermore, experiments are performed on two actuators connected in series to form different simple shapes. Finally, conclusions are drawn from the results, with the research outcomes listed and future work and improvements proposed.

\section{Methodology}

\subsection{Mathematical Model}

The governing equations used in the mathematical model are as follow. Firstly, the pressure applied on the extremity surfaces of the air chamber $(P)$ and the cross-section areas $\left(A_{\text {chamber }}\right)$ are computed:

$$
\begin{aligned}
& F_{p}=P A_{\text {chamber }}, \\
& F_{p}=F_{S M P 1}+F_{S M P 2}+F_{\text {sil }},
\end{aligned}
$$

where the force can be described by Hooke's law

$$
F_{\text {material }}=\epsilon_{\text {material }} E_{\text {material }} A_{\text {material }},
$$

where $\epsilon_{\text {material }}, E_{\text {material }}$, and $A_{\text {material }}$ are the strain, elastic modulus, and cross sectional area of the material respectively. We further assume that

$$
\epsilon_{\text {silicone }}=\frac{\epsilon_{S M P 1}+\epsilon_{S M P 2}}{2},
$$




$$
\begin{aligned}
& F_{S M P 1}=F_{S M P 2}, \\
& A_{S M P 1}=A_{S M P 2}=A_{S M P},
\end{aligned}
$$

then

$$
\epsilon_{S M P 2}=\frac{\epsilon_{S M P 1} E_{S M P 1}}{E_{S M P 2}} .
$$

Substituting (4) back to (2) and solve for $\epsilon_{S M P 1}$ we get

$$
\epsilon_{S M P 1}=\frac{F_{p}}{2 E_{S M P 1} A_{S M P}+0.5 A_{s i l} E_{s i l}\left(1+\frac{E_{S M P 1}}{E_{S M P 2}}\right)} .
$$

Bending angle can then be calculated as

$$
\theta=\frac{l_{S M P 1}-l_{S M P 2}}{h}=\frac{l_{0}\left(\epsilon_{S M P 1}-\epsilon_{S M P 2}\right)}{h},
$$

where $l_{0}$ is the initial length of the shape memory polymer layer, the strain of the adjustable stiffness composite layers $\epsilon_{S M P 1}, \epsilon_{S M P 2}$, and distance $(h)$ between the SMP layers (see figure 1(a)). Suppose that we consider the SMP1 to be the hot segment and SMP2 to be the cold segment then we have

$$
\theta=\frac{l_{\text {hot }}-l_{\text {cold }}}{h}=\frac{l_{0}\left(\epsilon_{\text {hot }}-\epsilon_{\text {cold }}\right)}{h} .
$$

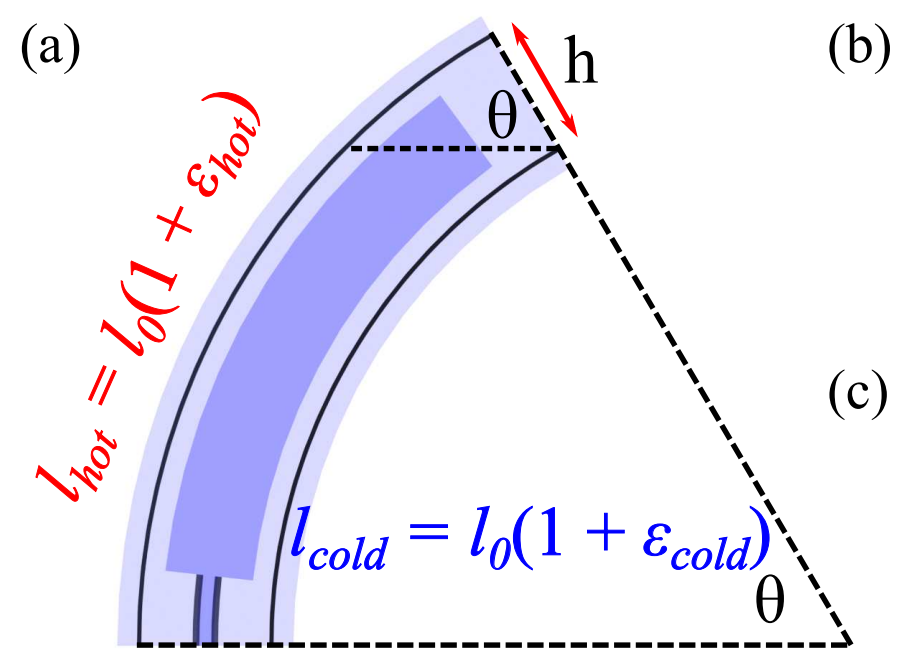

(b)

$$
h_{\text {actuator }}=w_{\text {actuator }}
$$

Figure 1. (a) Schematic of bent actuator, (b) cross section view of the actuator, (c) free-body diagram of element near the cap of the actuator.

We used $125 \mathrm{kPa}$ for the Young's modulus value for the Ecoflex-30 silicone rubber as reported in [30]. 
Table 1. Summary of actuator dimensions

\begin{tabular}{ll}
\hline Actuator element & Dimension \\
\hline SMP + conductive layers & $16 \mathrm{~mm}$ \\
Width $\left(w_{\text {SMP }}\right)$ & $60 \mathrm{~mm}$ \\
Length $\left(l_{0}\right)$ & $350 \mu \mathrm{m}$ \\
SMP thickness $\left(t_{S M P}\right)$ & $150 \mu \mathrm{m}$ \\
Conductive layer thickness $\left(t_{\text {cond }}\right)$ & $0.5 \mathrm{~mm}$ \\
SMP + Conductive layer thickness $\left(t_{\text {composite }}\right)$ & \\
\hline Silicone case & $20 \mathrm{~mm}$ \\
Width $\left(w_{\text {actuator }}\right)$ & $20 \mathrm{~mm}$ \\
Height $\left(h_{\text {actuator }}\right)$ & $76 \mathrm{~mm}$ \\
Length $\left(l_{\text {actuator }}\right)$ & \\
\hline Air chamber & $10 \mathrm{~mm}$ \\
Width $\left(w_{\text {chamber }}\right)$ & $10 \mathrm{~mm}$ \\
Height $\left(h_{\text {chamber }}\right)$ & $60 \mathrm{~mm}$ \\
Length $\left(l_{\text {chamber }}\right)$ & \\
\hline Actuator & $33 \mathrm{~mm}$ \\
Width $\left(w_{\text {total }}\right)$ & $33 \mathrm{~mm}$ \\
Height $\left(h_{\text {total }}\right)$ & $112 \mathrm{~mm}$ \\
Length $\left(l_{\text {total }}\right)$ & \\
\hline
\end{tabular}

\subsection{Design}

Following the mathematical model derived above we determined the appropriate dimensions for the actuator, as done in our previous work [8]. The dimensions are then calculated by substituting maximum desired bending angle $\theta_{\text {goal }}$ into (7). The maximum strain difference between the hot and cold segments is kept at $30 \%$ and the maximum desired bending angle is $\pi / 2$. The height of the chamber which affects the distance between the SMP layers, is an open parameter since we do not have any force-torque requirement of the actuator. However, based on previous experience and past experiments we found that lower limits for wall thickness of the actuator and the chamber height before bursting to be

$$
\begin{aligned}
& t_{\text {wall }} \geq 4 \mathrm{~mm}, \\
& h_{\text {chamber }} \geq 2 t_{\text {wall }} .
\end{aligned}
$$

Based on these, we designed the chamber height to be $10 \mathrm{~mm}$ and the distance between the SMP layers to be $16 \mathrm{~mm}$. Table 1 summarizes all the dimensions of all the elements of the proposed soft pneumatic actuator, also shown in figure 1.

\subsection{Fabrication}

2.3.1. Shape Memory Polymer One SMP substrate of $350 \mu \mathrm{m}$ thickness was obtained using approximately 12 grams of shape memory polymer pellets, SMP 3520 (SMP 
Technologies Inc., JP), with glass transition temperature of $35^{\circ} \mathrm{C}$. The pellets were placed at the center of steel plates covered with polytetrafluoroethylene (PTFE) impregnated fabrics. The fabric was used to prevent the SMP layers sticking to the metal plates and to avoid air inclusion in the resulting layer. Four polyimide (Kapton) shims of $350 \mu \mathrm{m}$ thickness were placed in the corners to obtain more uniform SMP sheet thickness (see figure 2(a)). The polymer pellets were left to melt in a heat press at low pressure for ten minutes near their melting temperature $\left(160^{\circ} \mathrm{C}\right)$, before higher pressure was gradually applied. The pressure reading on the heat press decreased as the pellets turned into a sheet. The desired thickness was obtained when the pressure reached steady state. After removal of the heat plates from the press, the SMP layer was left to cool down at room temperature ensuring its original configuration as flat as possible. Finally, the SMP layer was safely removed from the heat plates and was ready to use (figure $2(\mathrm{~b})$ ).

2.3.2. Flexible Heater The conductive paste was prepared using the following ingredients. The SIS (Polystyrene-polyisoprene-polystyrene) polymer (Sigma-Aldrich, USA) of $25 \%$ by weight was dissolved in $75 \%$ by weight DPGDME (dipropylene glycol dimethyl ether) solvent (Sigma-Aldrich, USA) until a homogeneous clear solution is obtained (about 2 hours or so depending on the amount). A magnetic stirrer was used to help with the initial mixing. Once a homogeneous solution was obtained, we then introduced the carbon black filler particles (Ketjenblack EC600, AkzoNobel, Netherlands), $6.8 \%$ of total volume, into the solution. In this work a density of 2.0 $\mathrm{g} / \mathrm{cm}^{3}$ was selected as the "true" density of the carbon black particles for calculating their volume fraction within the polymer matrix. However, it is important to note that this value is somewhat ill-defined and varies for different carbon black grades and, in practice, not all the free volume is wetted by liquids [37]. The solution and the filler were first manually mixed into a paste form before subsequently passed through a three-roll mill (EXAKT 50, EXAKT, Germany). The conductive paste was then deposited onto the SMP sheet using a film/layer applicator (ZUA2000, Zehntner, Switzerland). Two layers of $75 \mu \mathrm{m}$ each were deposited to the SMP substrate with at least two hours waiting in between (figure 2(c),(d)). The samples would roll up during the drying process so they need to be flattened by putting them on a hot plate $\left(70^{\circ} \mathrm{C}\right)$ and placing a metal plate on top to help press the samples. The samples were removed from the heat after one or two minutes and were cooled while leaving the metal plate pressing against the samples. The second conductive layer was then deposited to the flat samples with perpendicular direction from the first layer (figure $2(\mathrm{~d})$ ). Once two layers were deposited, the sample was then left for overnight drying.

The SMP-flexible heater composite was cut to shape using a laser cutter (Zing 40W, Epilog, USA). The specifications and dimensions of the SMP layers are described in figure 2(e). The active area of the SMP layers measures $60 \times 16 \mathrm{~mm}^{2}$. The active areas were bounded by 28 AWG polyurethane coated copper wires, which were bonded to the heating layer with conductive silver epoxy (CW2400, Chemtronics, USA). Temperature 


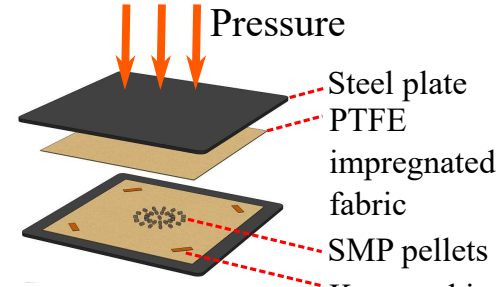

(a)

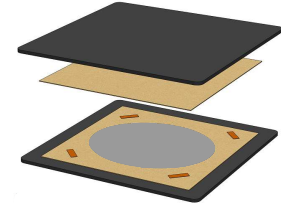

(b)

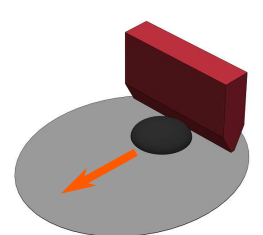

(c)

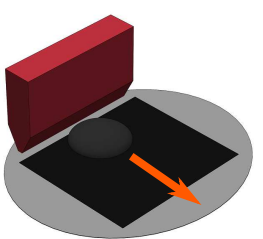

(d)

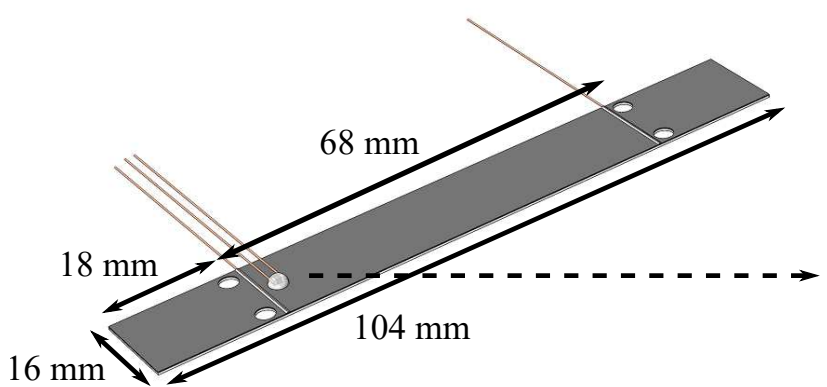

(e)

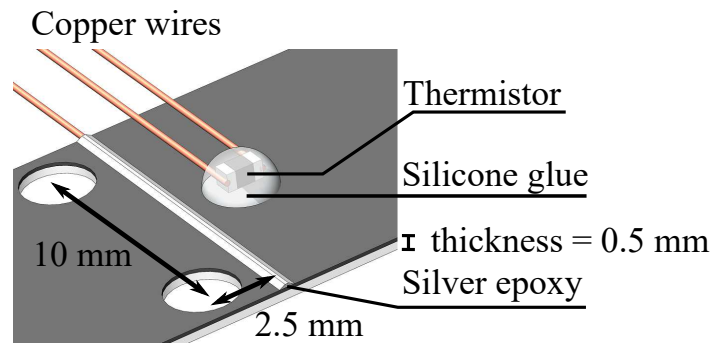

(f)

Figure 2. (a) Shape memory polymer (SMP) pellets are sandwiched between two polytetrafluoroethylene (PTFE) impregnated fabrics and two steel plates, polyimide (Kapton) shims are used to control the thickness of the formed SMP sheet, (b) SMP sheet are obtained after the pellets are reformed using a heat-press, (c) carbon black based conductive paste is deposited onto SMP sheet using a film applicator, (d) second layer of conductive layer is deposited with orthogonal direction from the first layer after at least two hour drying, (e) dimensions of the SMP-flexible heater composite cut to size using a laser cutter, (f) close up view of SMP-flexible heater components.

feedback of the SMP layers was done using thermistors that are placed near the end of each segment. In order to keep the thermistors in place on the SMP layer, a small amount of silicone glue (Dow Corning 732, Dow Corning, USA) was used (figure 2(f)). Once these steps have been successfully completed, the SMP layers were ready for use in the next fabrication steps.

Current flows through copper wires that are connected to $\mathrm{VCC}=40 \mathrm{~V}$ on one end of the heating layer towards the ground potential $=0 \mathrm{~V}$ on the other end. The start and stop of the current flow are controlled by a transistor connected to the power source. Each thermistor for temperature measurement of the heating layers was connected to a custom controller board. In order to maximize the overall solidity of the actuator, and to keep the silicone and SMPs elongating the same amount, adherence holes were cut in the layers, outside of the active areas. As further detailed in the next subsection, four aluminum rods were fitted through the silicone chamber and both of the SMP layers, as well as the 3D-printed caps.

\subsubsection{Actuator assembly Molding steps: The air chamber of the actuator was made} by creating a $10 \times 10 \times 60 \mathrm{~mm}^{3}$ wax core with a 3D-printed mold. The wax core was embedded into the silicone, before it was melted and extracted at the end of the fabrication of the actuator. Because of shrinking, it may be necessary to pour the 
(a)
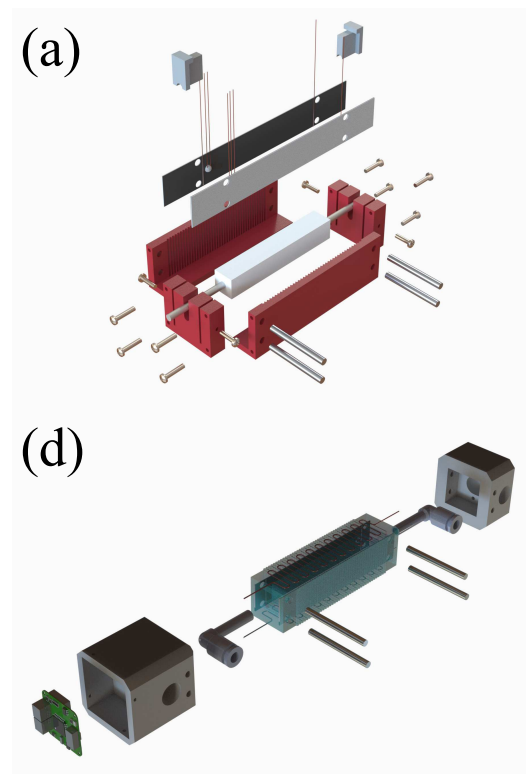

(b)

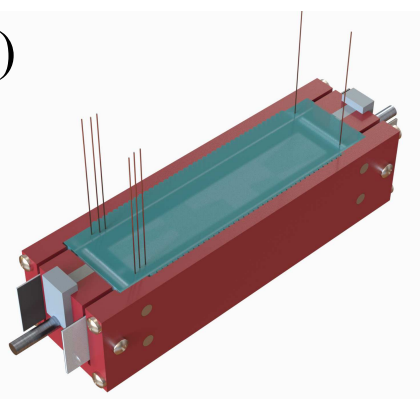

(e)

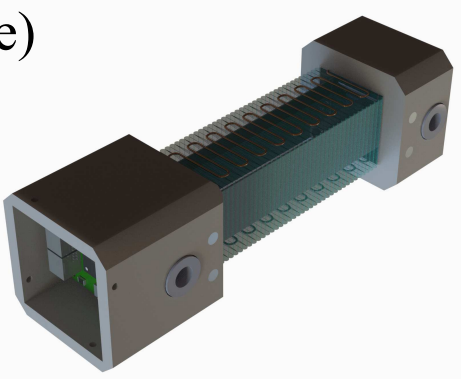

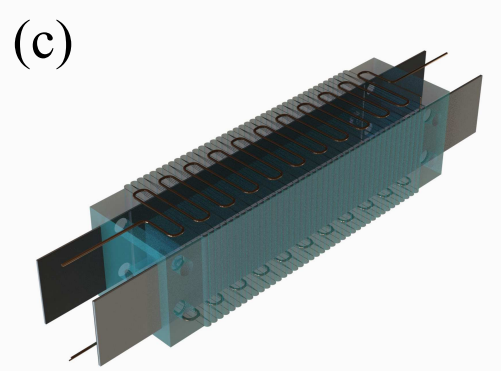

(f)

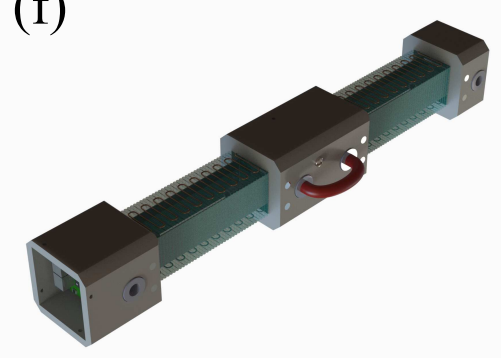

Figure 3. (a) exploded view of the mold assembly, wax core, and adjustable stiffness layer, (b) assembled mold and silicone poured into it, (c) cured pneumatic actuator with adjustable stiffness layer and electrical wires routed in serpentine path, (d) assembly of one pneumatic module with 3D-printed connectors and controller board, (e) assembled module, (f) assembled multi modules.

molten wax into the mold multiple times before achieving acceptable results. Once the air chamber negative was created, the main mold for the actuator was assembled. The main elements of the mold are screwed together in order to increase the geometric precision of the final actuator. Two slits of $0.5 \mathrm{~mm}$, located on each side of the mold, allowed the SMP layers to be held in the correct position along the width of the actuator throughout the fabrication. Four transversal aluminum rods of $3 \mathrm{~mm}$ diameter, allow the precise positioning of the SMP layers along the length of the actuator. Furthermore, four additional screws mounted laterally were used to keep the SMP layers stretched thus guarantees that they remain vertical during the molding process. Finally, the air chamber negative was inserted into the mold from the top, and secured in place with two printed parts that also stopped the silicone from overflowing when it was being poured (figure 3(a)).

After the main mold has been assembled, with each component secured in its correct position, Ecoflex 00-30 (Smooth-On Inc., USA) silicone was then poured into it with 1:1 ratio of part A and part B. About $40 \mathrm{~g}$ of silicone was needed to fill the mold. The silicone was then left curing overnight at room temperature (figure 3(b)). Once fully cured, the silicone body was then demolded by dissasembling the mold and removing the transversal aluminum rods. In order to constrain the radial expansion of the actuator when pressure is applied in the air chamber, the demolded silicone body was wrapped with nylon thread following small corrugations on the actuator walls. Moreover, the copper wires were guided following a serpentine path (figure $3(\mathrm{c})$ ). The air chamber 
negative was removed by heating the actuator in an oven and allowing the melting wax to leak out of the chamber.

Two push-in fittings (KQ2L23-04, SMC Pneumatics, USA) were inserted into 3Dprinted male and female connectors that would also house the custom printed circuit board. After having applied silicone glue on the inside faces of the connectors, they were then inserted at each extremity of the actuator by going through channels previously created by the wax core. Finally, four previously removed aluminum rods were inserted back into the actuator, through the connection modules (figure $3(\mathrm{~d})$ ). The silicone glue was left to cure overnight for full adhesive strength. Two independent pneumatic actuator modules can be easily connected in series (figure 3(f)) by securing the male and female connectors of subsequent modules with screws and connecting the push-in fittings with an air tube.

\subsection{Control board - architecture}

In order to achieve modularity through distributed control, we equip each actuator with a controller board. The controller board is a custom made printed circuit board (PCB) which contains 8-bit microcontroller to handle communication between modules and achieve closed-loop control of SMP temperature. The communication between the modules will be done through $\mathrm{I} 2 \mathrm{C}$ protocol and up to 127 modules can be connected with only two wires. To measure the temperature of the SMP layers, we utilize thermistors attached to them. Since the control board is not capable of directly reading the resistance of each thermistor, voltage divider circuits were used to compute the resistances indirectly. With the input voltage of each parallel circuit being known $(5 \mathrm{~V})$ and the voltage on a known resistor $(10 \mathrm{k} \Omega$ in our case) being also measured, the resistance of the thermistor can easily be computed using simple voltage divider equations. The current sources are the final step towards the powering of the SMP heating layers. The Xantrex XFR100-12 DC power supply was used to power both the slave controllers and heating layers of the actuators. Two transistors are used as switches to independently control the flow of current in the heating layers (2 layers for each actuator). An external Arduino board is used to interface the controller boards with a desktop computer which then used to log the measurement data. The Arduino board is also responsible to control a pressure valve as the (single) input for the actuators. The complete system architecture is shown in figure 4.

\subsection{Experimental Setup}

2.5.1. Silicone-SMP-flexible heater composite characterization In order to verify the previously derived mathematical model of the multi-DoF pneumatic actuator, the material characterization of the silicone, shape memory polymer, and flexible heater composite must be conducted. The SMP-flexible heater composite was laser cut to size according to the dimensions shown in figure 5(a). Two copper wires were affixed to the heating layer with silver epoxy. The composite was then embedded into Ecoflex 


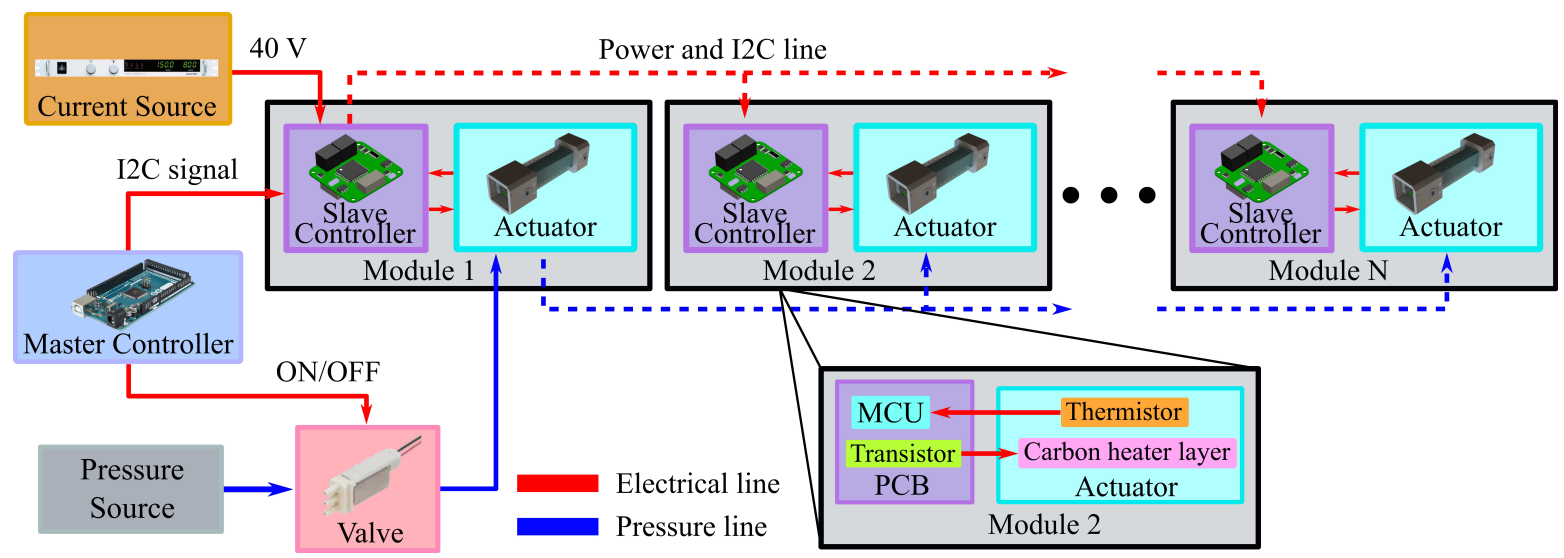

Figure 4. System architecture of $\mathrm{N}$ modules connected in series. (inset) functional block diagram of a module.

(a)

$$
\mathrm{L}_{0}=\stackrel{15 \mathrm{~mm}}{\longrightarrow}
$$

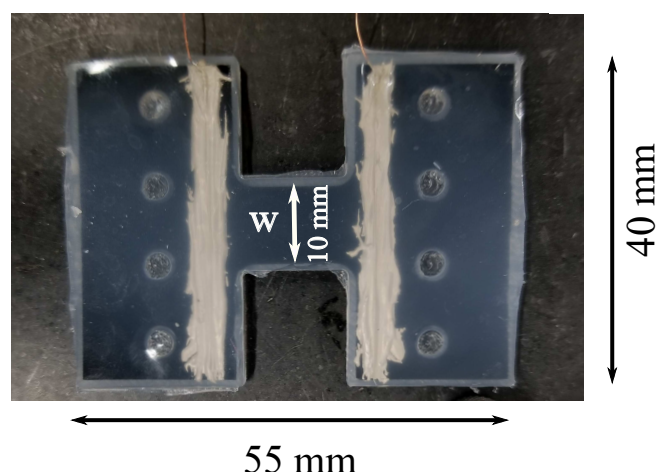

$55 \mathrm{~mm}$

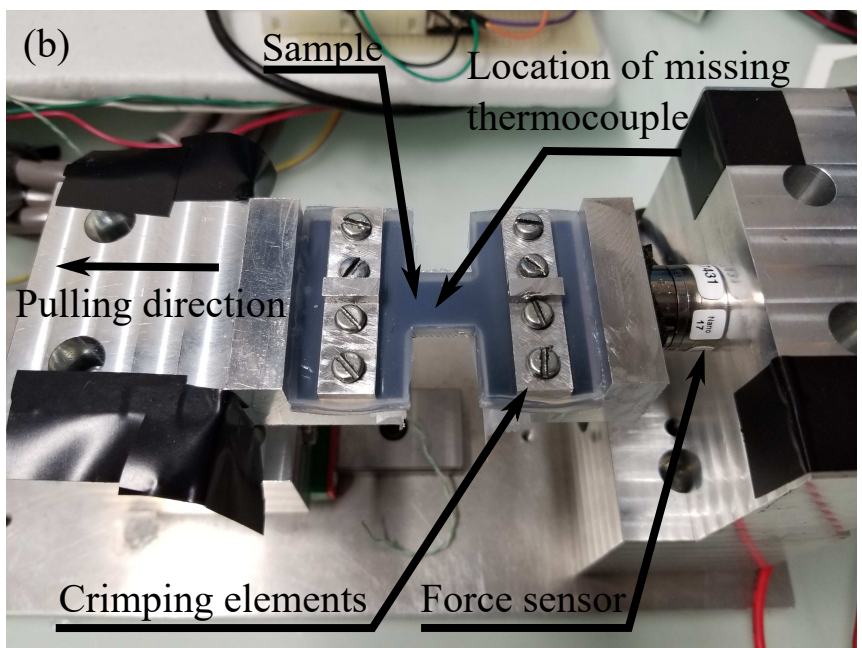

Figure 5. (a) Dimensions of the dogbone composite sample with copper wires affixed using silver epoxy, (b) stress-strain measurement setup with motorized linear stage and force sensor fix on one end. The temperature of the composite was measured using a thermocouple with only its location shown in the figure. The temperature reading was used as a feedback to control the sample temperature while it was being stretched.

00-30 silicone with sample thickness of $2.5 \mathrm{~mm}$. The stiffness tests of the SMP and silicone samples were conducted using a standard setup developed in the laboratory (figure 5(b)). This setup was comprised of a load cell on which one side of the sample was fixed and a mobile fixation system that allowed the sample to be stretched. While the sample was slowly stretched at a rate of $0.1 \mathrm{~mm} / \mathrm{s}$, the force corresponding to the displacement was recorded, which provides the data to plot a stress-strain curve. We repeated the loading and unloading cycles five times each for a range of temperature values investigated $\left(30-55^{\circ} \mathrm{C}\right)$ with $5^{\circ} \mathrm{C}$ increments.

The stress-strain plot was obtained by normalizing the displacement values by initial length $L_{0}$ and the force values by the area $\mathrm{A}=11.3 \times 2.5 \mathrm{~mm}^{2}$ of the cross section of the sample. The Young's modulus of the sample at different temperatures was obtained 
by linearly interpolating each curve and computing the slope of each interpolation.

2.5.2. Heating layer temperature uniformity test In order to evaluate the uniformity of the heater layer, we observed an activated SMP sample with heating layer under an infra-red camera. The heater was powered by a $50 \mathrm{~V}$ source while keeping the maximum electrical current of $0.1 \mathrm{~A}$. No temperature control was implemented in this experiment. The activated sample was also strained to see whether straining the sample would introduce hot spots. Hot spots are not desirable as they can create localized heating and melt the SMP layer.

2.5.3. Single module characterization test We conducted bending angle measurements for the range of SMP temperatures. For this experiment, we maintained one SMP layer to be at $30^{\circ} \mathrm{C}$ while increasing the temperature of the other SMP layer until $45^{\circ} \mathrm{C}$ with $5^{\circ} \mathrm{C}$ increments. When the desired temperatures were reached we supplied pressurized air to the module hence creating bending motion which repeated for three times. Pressurized air of 10, 11, and 12 psi was used to achieve the bending motion. The bending angle was measured using the OptiTrack motion tracking system (NaturalPoint Inc., USA) with IR reflective markers placed on top of the actuator. We fabricated three modules and repeated the experiments for all of them in order to evaluate the repeatability of our fabrication method.

In order to evaluate the hyper-elastic behavior of the proposed actuator, we conducted experiments at which the bending angle was measured as the actuator was incrementally pressurized and un-pressurized. A total of four experiments were conducted at which we heated one side of the actuator from $30^{\circ} \mathrm{C}$ up to $45^{\circ} \mathrm{C}$ while keeping the other side constant at $30^{\circ} \mathrm{C}$. For each desired temperature pair, the actuator was incrementally pressurized up to 12 psi $(0,2,4,6,8,10,11,12$ psi) with about 15 seconds delay in between the pressure values. The time delay was important to let the actuator to settle and reach steady state before the bending angle was recorded. Once maximum pressure of 12 psi was reached, the actuator was then un-pressurized following the same pressure values as before. We also conducted measurements on the blocking force capabilities of the bending actuators. In this experiment, the distal end of the actuator was fixed onto a load cell while the proximal end of the actuator and the load cell were secured using vises. The experiment was done with three different actuators over three different input pressure values (10-12 psi).

2.5.4. Multi-module test - shape forming To evaluate the feasibility of the modular approach of our proposed actuator, we connected two modules in series and controlled each module independently to achieve different shapes. Through this experiment we demonstrated actuator motions that normally cannot be achieved without a multichamber and/or multi-valve system. In this paper, we simplified the experiment for a planar case. Each module has 4 realizable states defined by the temperature of the embedded SMP composites: left segment cold right segment hot, left segment hot right 


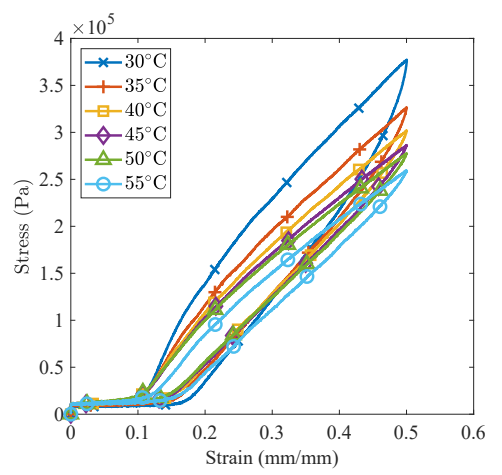

(a)

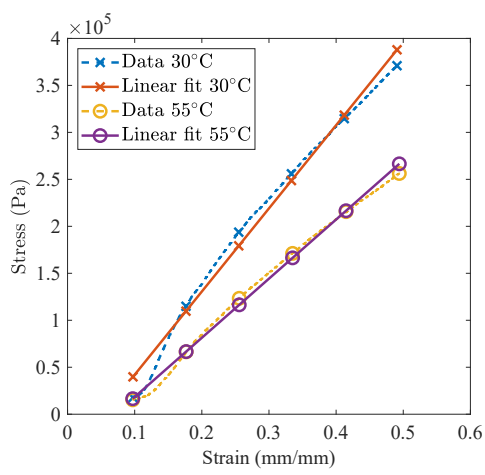

(b)

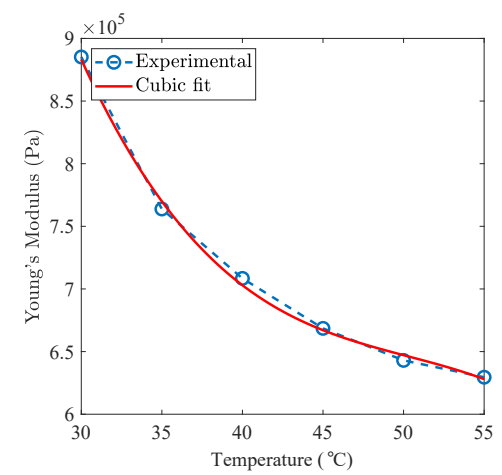

(c)

Figure 6. (a) Stress-strain curve of the SMP-flexible heater composite under different temperatures, (b) linear fit of the stress-strain curve to approximate the Young's modulus of the composite under loading for minimum and maximum tested temperatures, (c) Young's modulus as a function of temperature with cubic fit.

segment cold, both segments hot, both segments cold. By combining these possible states we could create different interesting shapes.

\section{Results}

\subsection{Stiffness vs Temperature}

The uniaxial loading of the SMP sheet embedded in silicone showed a decrease in stiffness as temperature increased (shown in figure 6(a)). For each different temperature we loaded and unloaded the composite sample for 5 times. Since the results for all the five cycles were consistent, only results for the last cycle were plotted and used to determine the Young's modulus.

Maximum stiffness change of 1.4 times were observed between the minimum and maximum temperatures of $30^{\circ} \mathrm{C}$ and $55^{\circ} \mathrm{C}$. We also plotted the variation of Young's modulus as a function of temperature and a third order polynomial function was fitted to the curve given as follows

$$
E_{\text {comp }}=-20.2 T^{3}+3047 T^{2}-1.56510^{5} T+3.38310^{6},
$$

where $E_{\text {comp }}$ is the effective elastic modulus of the composite and $T$ is the temperature of the composite. Furthermore, considering the Hooke's law for the composite sample, we have

$$
\begin{aligned}
\epsilon_{\text {comp }} E_{\text {comp }} A_{\text {comp }} & =\epsilon_{\text {sil }} E_{\text {sil }} A_{\text {sil }} \\
& +\epsilon_{S M P} E_{S M P} A_{S M P .} .
\end{aligned}
$$

Solving for $E_{S M P}$ we get

$$
E_{S M P}=\frac{E_{\text {comp }} A_{\text {comp }}-E_{\text {sil }} A_{\text {sil }}}{A_{S M P}} .
$$

We can then substitute (10) back to (4) and (5), and finally calculate the bending angle using (6) given pressure and temperature inputs. 

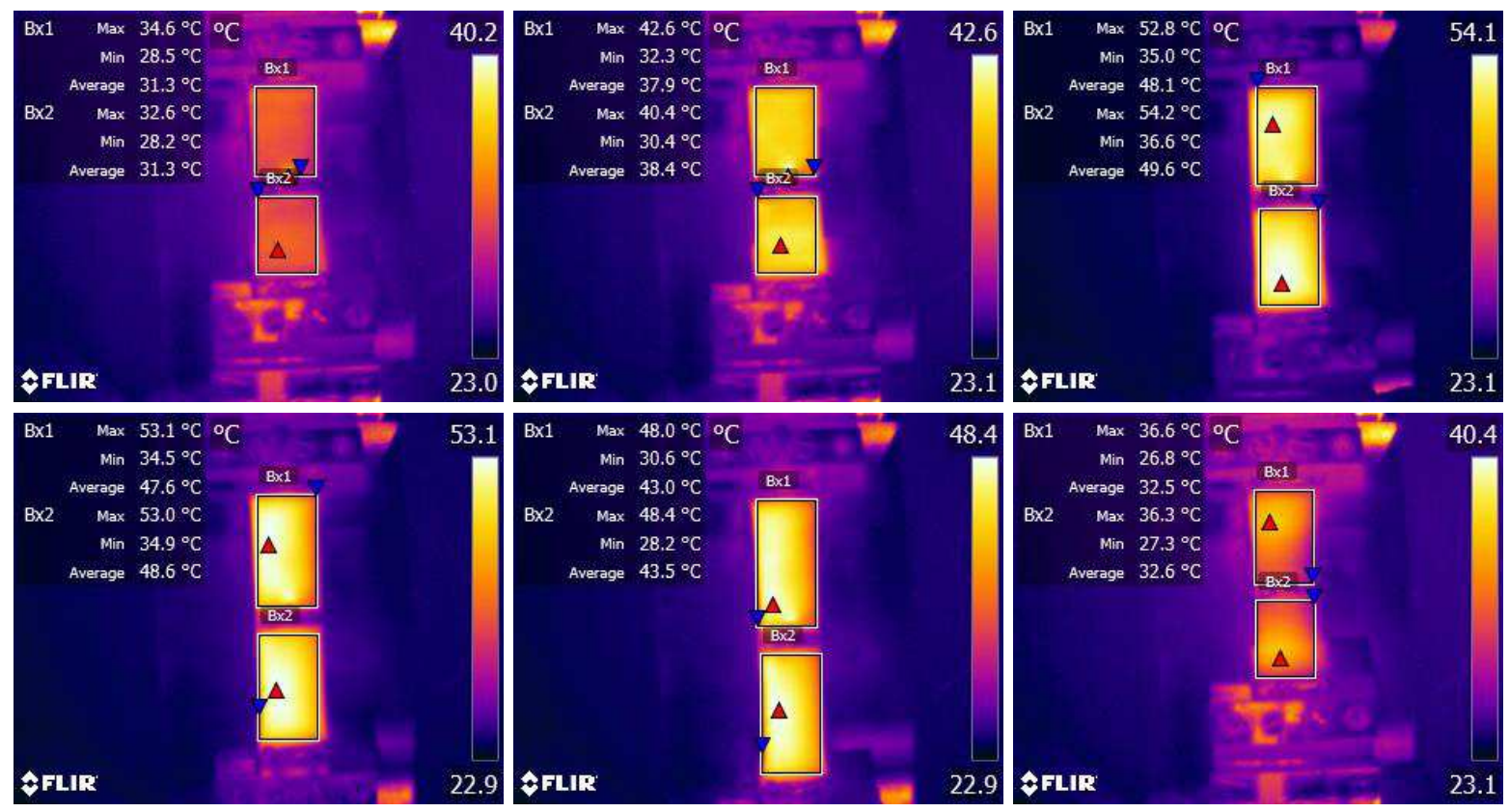

Figure 7. Thermal camera data of a segmented SMP-flexible heater composite being stretched up to $50 \%$ strain and un-stretched back to original length. Uniform heating and no hot-spots are observed when the heater was powered by a $50 \mathrm{~V}$ source.

\subsection{Temperature uniformity}

From the result shown in figure 7 , we can see that the heating profile is uniform despite the small gradient and no hotspots were observed. Non-uniform heating would result in localized stiffness change that is not desirable. Furthermore, localized heating may introduce cracks to the SMP layer which can lead to eventual burning of the layer. Uniform heating along both the heater's length and width allows us to place the temperature sensor near the edge of the module. This will also minimize the length of copper wires need to be routed in serpentine path.

\subsection{Single module characterization results}

Figure 8 shows a single module being actuated with 12 psi pressurized air while the right segment was kept at $30^{\circ} \mathrm{C}$ and the left segment was incrementally heated to $45^{\circ} \mathrm{C}$. As expected, the heated SMP segment elongates, allowing the actuator to bend to the right.

We also recorded the measurements for segment temperature and bending angle as shown in figure 9. We can see that the hot side of the actuator was able to reach the desired temperature although the cold side was slightly affected by the hot side. Even though silicone rubber is a great heat insulator, this was attributed to heating over a long period of time. So there is a need to decrease the resistance of the heating layer, possibly by increasing the number of conductive layers. However, the temperature increase was only about $2.5^{\circ} \mathrm{C}$ and the temperature was still below the glass transition 

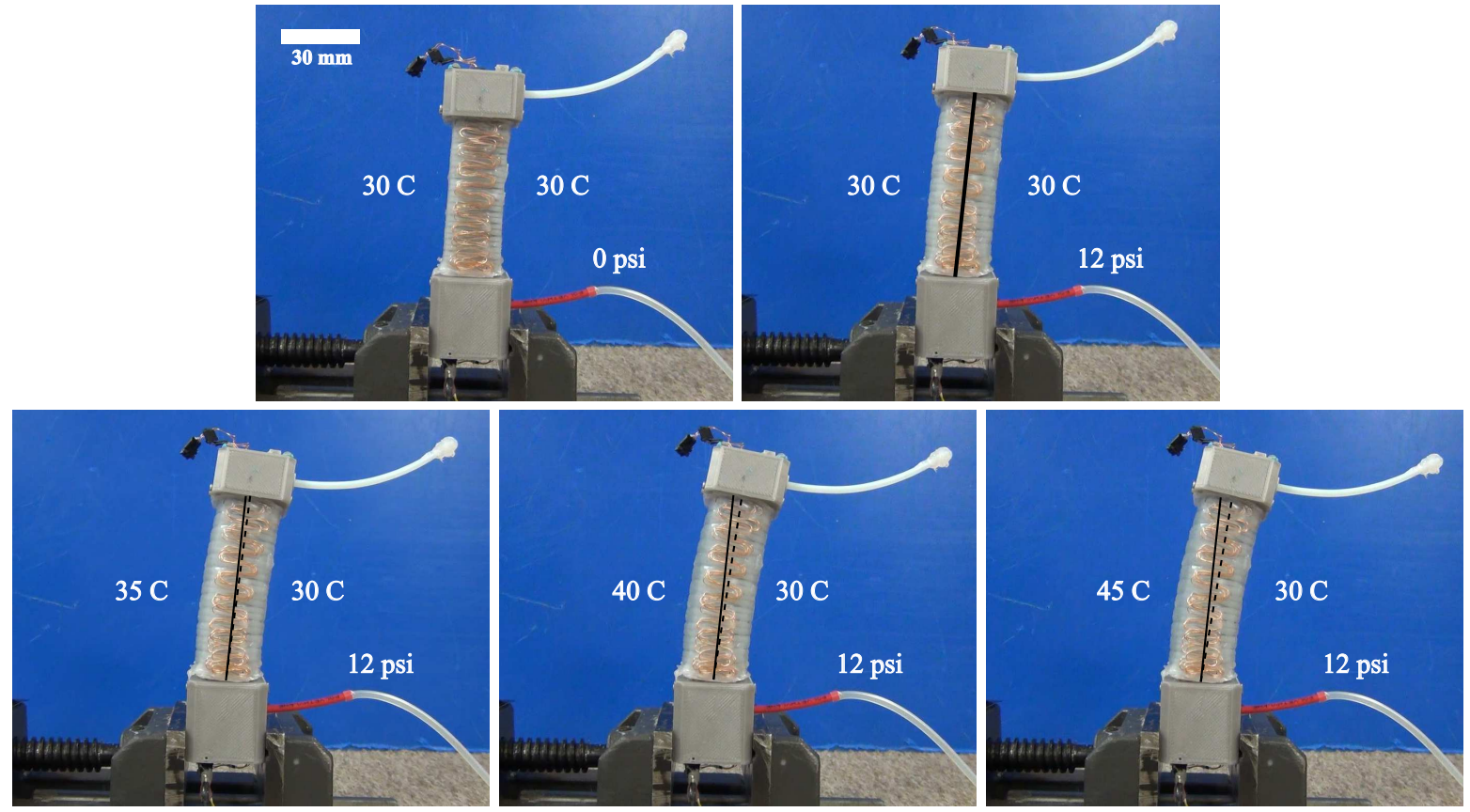

Figure 8. Snapshot of single module actuated with 12 psi pressurized air with the right segment of the module kept at $30^{\circ} \mathrm{C}$ (cold side) and the left segment is incrementally increase to $45^{\circ} \mathrm{C}$ (hot side).

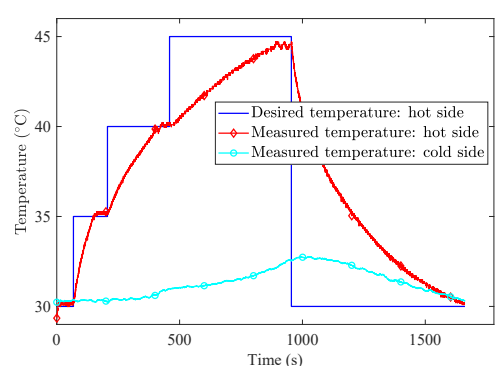

(a)

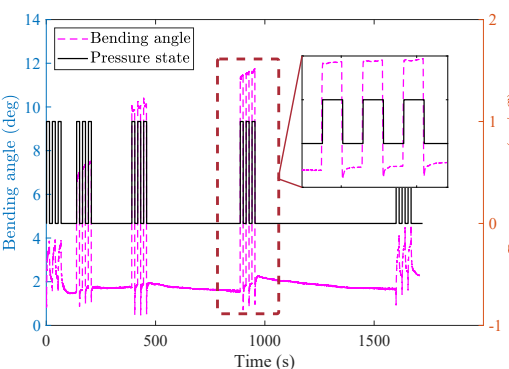

(b)

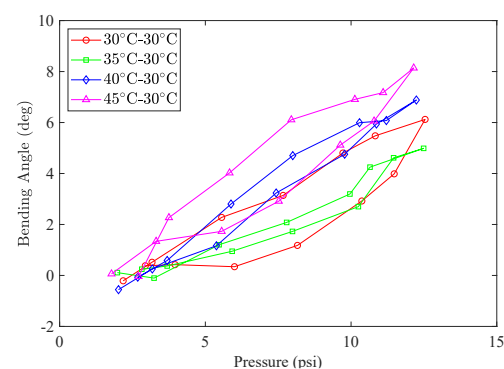

(c)

Figure 9. (a) Temperature response of the hot and cold segments of the adjustablestiffness actuator measured using embedded thermistors. (b) Bending angle response of the adjustable-stiffness soft actuator synchronized to the temperature response data shown in (a). The actuator is pressurized (pressure state is equal to 1) and un-pressurized (pressure state is equal to 0 ) for three times whenever the desired temperatures of the actuator's hot and cold segments is achieved. Close up view of the bending angle response for hot segment temperature of $45^{\circ} \mathrm{C}$ and cold segment temperature of $30^{\circ} \mathrm{C}$ (inset). (c) Measured bending angle as a function of input pressure for loading and unloading sequences. Hysteresis can be observed despite being minimal.

temperature of the SMP.

Most if not all the temperature-dependent variable stiffness structures will be greatly influenced by the ambient temperature. However, for most cases the assumption of known constant ambient temperature is reasonable. All of our experiments were performed in an environmentally-controlled laboratory. Since the bandwidth of our 
heating layer at the moment is very low we decided not to incorporate a heat transfer model into our control system. Currently, our control system will output maximum electrical current it can provide to drive the heating layers to their desired temperature. In the future given heating layers with much higher bandwidth we can then incorporate a heat transfer model that will take into account the temperature of each heating layer and adjust the current appropriately so that the hotter side will give minimum impact on the colder side. Furthermore, we can also estimate the heating and cooling effect contributed from the environment by measuring the time needed by the heating layer to achieve a given temperature change. This can also be included into the model to modulate the driving current.

At room temperature, it took 8 seconds to increase the SMP's temperature from $30^{\circ} \mathrm{C}$ to $31^{\circ} \mathrm{C}$ and 74 seconds from $40^{\circ} \mathrm{C}$ to $41^{\circ} \mathrm{C}$ respectively (extracted from figure $9(\mathrm{~b})$ ). Due to resistive heating nature of the heating layer we expect the response time to increase as the temperature increases. This response time can be improved by reducing the resistance of the heating layer or increasing the driving voltage, which both will increase the amount of electrical current passing through the heating layer. The driving voltage was limited due to the fact that the same voltage line was used to power both the heating layer and the microcontroller board, which operates at lower voltage. Separate power lines for the controller board and heater current source can be done to mitigate this limitation. The actuator was pressurized and de-pressurized for three times whenever the desired temperatures of the segments were reached as shown in figure $9(\mathrm{~b})$. Moreover, the rise time of the actuator is about 1.1 seconds. From figure 9(c), we can observe the hysteresis behavior on the actuator, despite it being minimal. This is most likely caused by the relatively small strain induced by the pressure source. A maximum blocking force of $1.9 \mathrm{~N}$ at 12 psi was found between the three actuators analyzed in this paper. Larger pressure values would result in higher blocking force before the actuator bursting point.

The results for three fabricated modules are summarized in figure 10. Our model and experimental result match very well. As expected, the bending angle increased as the input pressure increased. However, we also observed significant variations between the modules, which may be attributed to fabrication uncertainties. It is clear that increasing the input pressure will lead to increase in bending angle, but this is not always possible since at higher pressure the silicone body may start to radially deform at places where the radial constraint is the weakest and may eventually burst. Looking at (6), one possible way to increase the bending angle is by reducing the distance between the SMP layers, this may be appropriate depending on the application of the proposed actuator. Another possible way is to increase the amount of stiffness change, which could be done by increasing the temperature difference or choosing another SMP material for the adjustable stiffness layers. 
1

2

3

4

5

6

7

8

10

11

12

13

14

15

16

17

18

19

20

21

22

23

24

25

26

27

28

29

30

31

32

33

34

35

36

37

38

39

40

41

42

43

44

45

46

47

48

49

50

51

52

53

54

55

56

57

58

59

60
Multi-DoF soft pneumatic actuator with adjustable stiffness layer

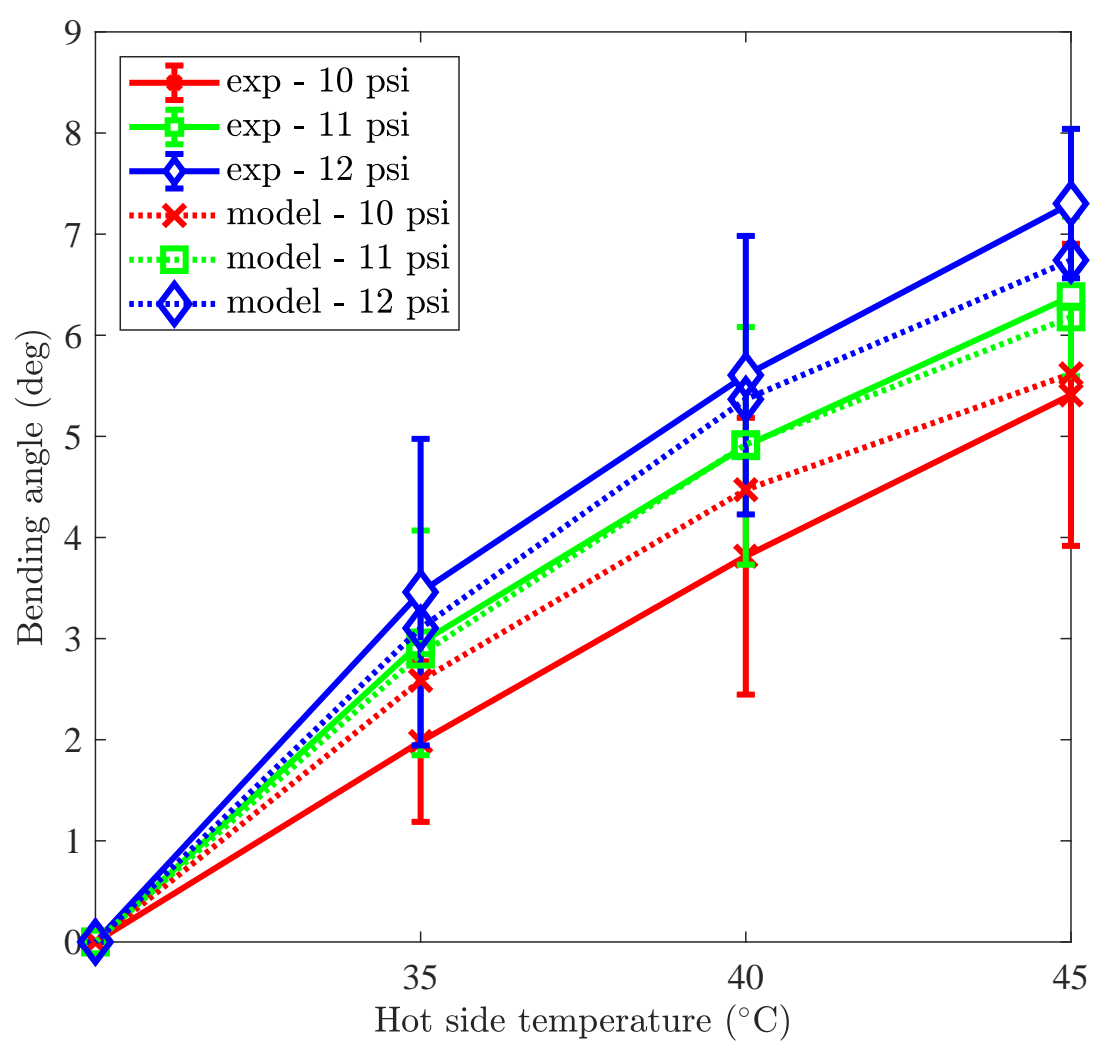

Figure 10. Comparison between the experimental data for three different modules and the mathematical model. The experimental data matches the model proposed. As the pressure increases the amount of bending angle increases. The temperature increase in hot side also corresponds to an increase in bending angle.

\subsection{Multi-module experiment}

Figure 11 demonstrates the feasibility of controlling the modules independently. We can achieve various complex shapes through combining four different states of each module. For example, by heating all the segments on one same side we can generate $\mathrm{C}$-shape configuration while heating opposite sides will result in S-shape. This normally would require the use of multi-chambers or multi-valves, however in our case only one pressure line was necessary and four electrical wires in total for power and communication lines up to 127 modules. Furthermore, the modules could lock their shape when cooled down to temperature below the transition temperature, after which point pressure may be removed without affecting the shape of the modules.

\section{Conclusion \& Future Work}

We proposed an adjustable stiffness pneumatic actuator utilizing a thermoplastic polyurethane shape memory polymer with a carbon black flexible heater. The adjustable stiffness layer is responsible for augmenting the degrees of freedom of the pneumatic actuator without the use of multi-source, multi-chamber, or multi-valve pressure 

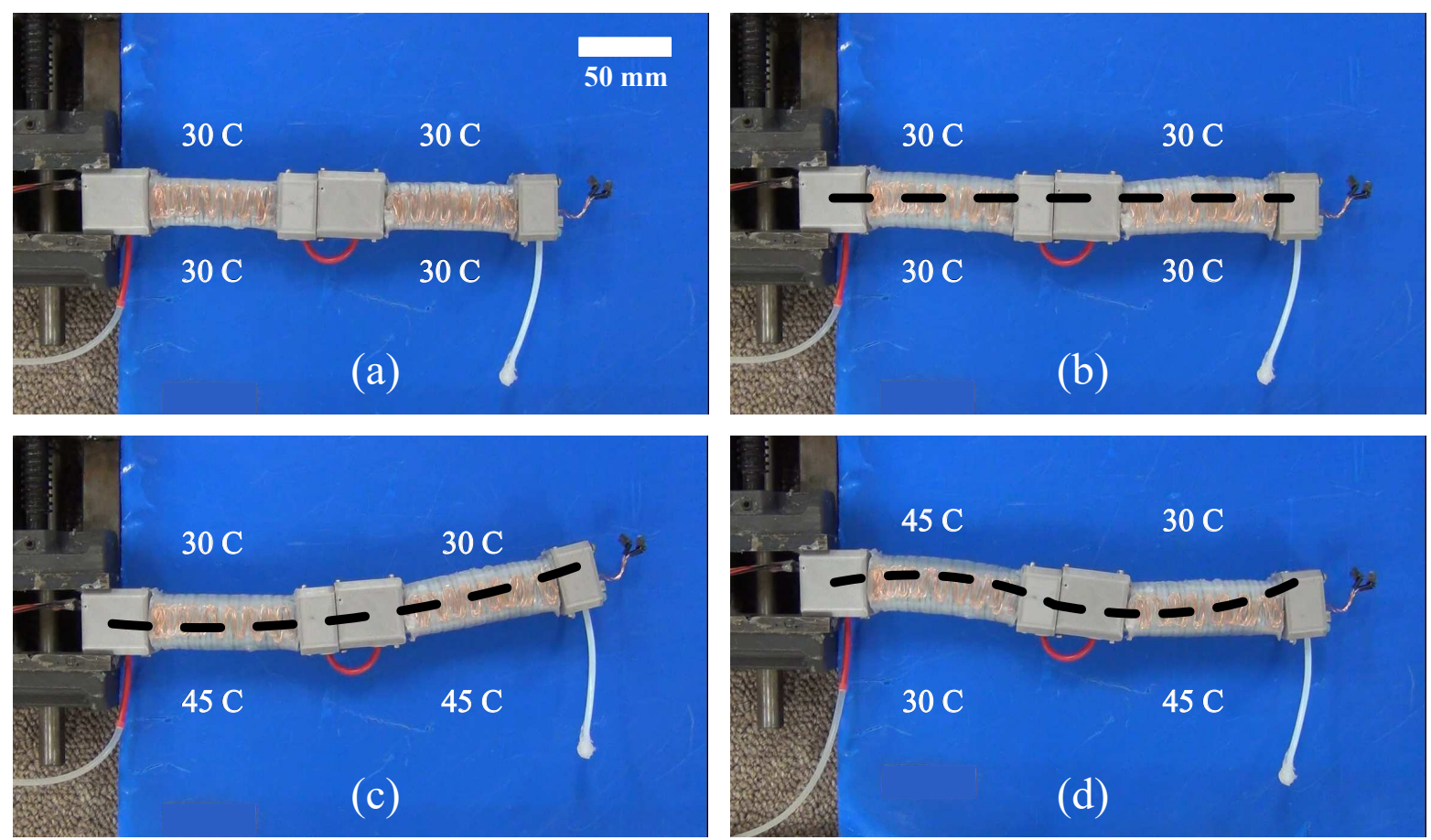

Figure 11. Snapshot of multi-module actuation forming different shapes (a) straight un-actuated, (b) straight actuated, (c) C-shape, (d) S-shape. The dashed line represents the goal configuration.

systems. Since the stiffness change is temperature-driven, the bandwidth is expected to be quite low. Such systems, however, would be very well suited for applications where quasi-static, or relatively slow, variation in stiffness is sufficient. The actuator is capable of generating a maximum 7-degree bending angle with the highest temperature of $45^{\circ} \mathrm{C}$ and $30^{\circ} \mathrm{C}$ for hot and cold sides respectively, and 12 psi input pressure.

The actuator bending angle can be improved by using tougher elastomer materials for fabrication since higher input pressure can be sustained by the actuators while providing higher strain to the SMP layers. Alternatively, a smaller diameter actuator would result in a higher bending angle for the same SMP properties and pressure, with a trade-off in smaller force output, as can be deduced from the mathematical model. Possible alternatives for the SMP materials and heating methods could be explored to increase the stiffness variation range. Nevertheless, we have demonstrated the benefit of our modular approach through independent activation of the actuation modules connected in series. The modular approach will be beneficial for scalability of the system without increasing complexity.

Our future work includes improving the performance of the adjustable stiffness layer. In order to achieve a faster heating time, thicker heating layers may be manufactured by depositing more conductive layers. Faster heating times will be beneficial for applications that require fast change of stiffness. The active degree of freedom of the pneumatic actuator can be further augmented by segmentation of the heater layer e.g. a two-segment layer can be achieved using two wires at the end of 
segment having low (ground) potential and another wire in the middle of the segment having high (VCC) potential. Each segment can then be activated independently, hence doubling the degrees-of-freedom from two to four per actuation module. Lastly, self-sensing capability of the actuator can be investigated by measuring the change in resistance as a function of stretching of the heating layer with a corresponding increase in manufacturing complexity.

\section{Acknowledgments}

This material is based upon work partially supported by the National Science Foundation (NSF) under Grant Nos. IIS-1551219, CMMI-1728412, and CMMI-1752195. Any opinions, findings, and conclusions or recommendations expressed in this material are those of the authors and do not necessarily reflect the views of the NSF.

\section{References}

[1] Connolly F, Polygerinos P, Walsh CJ and Bertoldi K 2015 Mechanical programming of soft actuators by varying fiber angle Soft Robot. 2 26-32

[2] Marchese A D, Katzschmann R K and Rus D 2015 A recipe for soft fluidic elastomer robots Soft Robot. 2 7-25

[3] Roche E T, Wohlfarth R, Overvelde J T, Vasilyev N V, Pigula F A, Mooney D J, Bertoldi K and Walsh C J 2014 A bioinspired soft actuated material Adv. Mater. 26 1200-6

[4] Polygerinos P, Wang Z, Galloway K C, Wood R J and Walsh C J 2015 Soft robotic glove for combined assistance and at-home rehabilitation Robot. Auton. Systs. 73 135-43

[5] Cianchetti M, Ranzani T, Gerboni G, Nanayakkara T, Althoefer K, Dasgupta P and Menciassi A,2014 Soft robotics technologies to address shortcomings in today's minimally invasive surgery: the STIFF-FLOP approach Soft Robot. 11(2) 122-31

[6] Tolley M T, Shepherd R F, Mosadegh B, Galloway K C, Wehner M, Karpelson M, Wood R J and Whitesides G M 2014 A resilient, untethered soft robot Soft Robot. 1 213-23

[7] Sanan S, Ornstein M H and Atkeson C G 2011 Physical human interaction for an inflatable manipulator Proc. IEEE Int. Conf. on Engineering in Medicine and Biology Society (EMBC '11) (Boston, MA, Aug. 2010) p 7401-4

[8] Firouzeh A, Salerno M and Paik J 2015 Soft pneumatic actuator with adjustable stiffness layers for multi-dof actuation Proc. IEEE/RSJ Int. Conf. on Intelligent Robots and Systems (IROS '15) (Hamburg, Germany, Sept. 2015) p 1117-24

[9] Rus D and Tolley M T 2015 Design, fabrication and control of soft robots Nature $\mathbf{5 2 1}$ 467-75

[10] Polygerinos P, Correll N, Morin S A, Mosadegh B, Onal C D, Petersen K, Cianchetti M, Tolley M T and Shepherd R F 2017 Soft robotics: Review of fluiddriven intrinsically soft devices; manufacturing, sensing, control, and applications in humanrobot interaction Adv. Eng. Mater. 191700016

[11] Suzumori K, Iikura S and Tanaka H 1991 Development of flexible microactuator and its applications to robotic mechanisms Proc. IEEE Int. Conf. on Robotics and Automation (ICRA '91) (Sacramento, California, April 1991) p 16227

[12] McMahan W, Chitrakaran V, Csencsits M, Dawson D, Walker I D, Jones B A, Pritts M, Dienno D, Grissom M and Rahn C D 2006 Field trials and testing of the OctArm continuum manipulator Proc. IEEE Int. Conf. on Robotics and Automation (ICRA '06) (Orlando, Florida, May 2006) p 2336-41

[13] Luo M, Pan Y, Skorina E H, Tao W, Chen F, Ozel S and Onal C D 2015 Slithering towards 
autonomy: a self-contained soft robotic snake platform with integrated curvature sensing Bioinspir. Biomim. 10055001

[14] Gandhi F and Kang S G 2007 Beams with controllable flexural stiffness Smart Mater. Struct. 16 1179-84

[15] McKnight G and Henry C 2005 Variable stiffness materials for reconfigurable surface applications Proc. SPIE 5761 Smart Structures and Materials 2005: Active Materials: Behavior and Mechanics (San Diego, California, May 2005) p 119-27

[16] Shintake J, Schubert B, Rosset S, Shea H and Floreano D 2015 Variable stiffness actuator for soft robotics using dielectric elastomer and low-melting-point alloy Proc. IEEE/RSJ Int. Conf. on Intelligent Robots and Systems (IROS '15) (Hamburg, Germany, Sept. 2015) p 1097-102

[17] Yang Y, Chen Y, Li Y, Chen M Z and Wei Y 2017 Bioinspired robotic fingers based on pneumatic actuator and 3D printing of smart material Soft Robot. 4 147-62

[18] Wei Y, Chen Y, Ren T, Chen Q, Yan C, Yang Y and Li Y 2016 A novel, variable stiffness robotic gripper based on integrated soft actuating and particle jamming Soft Robot. 3 134-43

[19] Pan Y, Luo M, Skorina E H and Onal C D 2016 Controlling the bending response of a multi-layer composite module Proc. Adhesion Society Annual Meeting

[20] Hinchet R, Vechev V, Shea H and Hilliges O 2018 DextrES: wearable haptic feedback for grasping in VR via a thin form-factor electrostatic brake Proc. The 31st Annual ACM Symposium on User Interface Software and Technology (Berlin, Germany, Oct. 2018) p 901-12

[21] Shan W, Diller S, Tutcuoglu A and Majidi C 2015 Rigidity-tuning conductive elastomer Smart Mater. Struct. 24065001

[22] Tonazzini A, Mintchev S, Schubert B, Mazzolai B, Shintake J and Floreano D 2016 Variable stiffness fiber with selfhealing capability Adv. Mater. 28 10142-148

[23] Shan W, Lu T and Majidi C 2013 Soft-matter composites with electrically tunable elastic rigidity Smart Mater. Struct. 22085005

[24] Fassler A and Majidi C 2015 Liquidphase metal inclusions for a conductive polymer composite Adv. Mater. 27 1928-32

[25] Guo C F and Ren Z 2015 Flexible transparent conductors based on metal nanowire networks Mater. Today 18 143-54

[26] Amjadi M, Yoon Y J and Park I 2015 Ultra-stretchable and skin-mountable strain sensors using carbon nanotubesecoflex nanocomposites Nanotechnology 26375501

[27] Yan C, Wang J, Kang W, Cui M, Wang X, Foo C Y, Chee K J and Lee P S 2014 Highly stretchable piezoresistive graphenenanocellulose nanopaper for strain sensors Adv. Mater. 26 2022-27

[28] Takashima K, Sugitani K, Morimoto N, Sakaguchi S, Noritsugu T and Mukai T 2014 Pneumatic artificial rubber muscle using shape-memory polymer sheet with embedded electrical heating wire Smart Mater. Struct. 23125005

[29] Flandin L, Chang A, Nazarenko S, Hiltner A and Baer E 2000 Effect of strain on the properties of an ethyleneoctene elastomer with conductive carbon fillers J. Appl. Polym. Sci. 76 894-905

[30] Park Y L, Majidi C, Kramer R, Brard P and Wood R J 2010 Hyperelastic pressure sensing with a liquid-embedded elastomer J. Micromech. Microeng. 20125029

[31] Onal C D and Rus D 2012 A modular approach to soft robots Proc. IEEE RAS 8 EMBS Int. Conf. on Biomedical Robotics and Biomechatronics (BioRob '12) (Rome, Italy, June 2012) p 1038-45

[32] Lee J Y, Kim W B, Choi W Y and Cho K J 2016 Soft robotic blocks: introducing SoBL, a fast-build modularized design block Robot. Autom. Mag. 23 30-41

[33] Skorina E H, Tao W, Chen F, Luo M, Onal C D Motion control of a soft-actuated modular manipulator Proc. IEEE Int. Conf. on Robotics and Automation (ICRA '16) (Stockholm, Sweden, May 2016) p 49975002

[34] Santoso J, Skorina E H, Luo M, Yan R and Onal C D 2017 Design and analysis of an origami continuum manipulation module with torsional strength Proc. IEEE/RSJ Int. Conf. on Intelligent Robots and Systems (IROS '17) (Vancouver, Canada, September 2017) p 2098104 
[35] Robertson M A and Paik J 2017 New soft robots really suck: Vacuum-powered systems empower diverse capabilities Sci. Robot. 2 eaan6357

[36] Natividad R, Del Rosario Jr M, Chen P C and Yeow C H 2018 A reconfigurable pneumatic bending actuator with replaceable inflation modules Soft Robot. 5 304-17

[37] Rossman R P and Smith W R 1943 Density of carbon black by helium displacement Ind. and Eng. Chem. 35 972-6 